\title{
Building a Competing Endogenous RNA Network to Find Potential Long Non-Coding RNA Biomarkers for Pheochromocytoma
}

\author{
Ying-Chun Liang Yu-Peng Wu Dong-Ning Chen Shao-Hao Chen \\ Xiao-Dong Li Xiong-Lin Sun Yong Wei Xu Ning Xue-Yi Xue \\ Department of Urology, First Affiliated Hospital of Fujian Medical University, China
}

Key Words

Competing endogenous RNA • Long non-coding RNA • Biomarker • Pheochromocytoma

\begin{abstract}
Background/Aims: Accumulating evidence has shown that long non-coding RNAs (IncRNAs) in competing endogenous RNA (ceRNA) networks play crucial roles in tumor survival and patient prognosis; however, studies investigating ceRNA networks in pheochromocytoma (PCC) are lacking. In this study, we investigated the pathogenesis of PCC and whether IncRNAs acting through ceRNAs networks were associated with prognosis. Methods: A total of 183 PCC samples and 3 control samples from The Cancer Genome Atlas database were analyzed. The Empirical Analysis of Digital Gene Expression Data package in R (edgeR) was used to analyze differentially expressed RNAs. Biological processes and pathways functional enrichment analysis were performed based on the Database for Annotation, Visualization, and Integrated Discovery (DAVID) database. LncRNA/mRNA/miRNA ceRNA network was constructed by Cytoscape v3.0 software based on the differentially expressed RNAs Survival package in R was used to perform survival analysis. Results: In total, 554 differentially expressed IncRNAs, 1775 mRNAs and 40 miRNAs were selected for further analysis. Subsequently, 23 IncRNAs, 22 mRNAs, and 6 miRNAs were included in the constructed ceRNA network. Meanwhile, two of the 23 IncRNAs (C9orf147 and BSN-AS2) were identified as independent predictors of overall survival in $\mathrm{PCC}$ patients $(\mathrm{P}<0.05)$. Conclusion: This study improves the understanding of IncRNA-related ceRNA networks in PCC and suggests that the IncRNAs C9orf147 and BSNAS2 could be independent prognostic biomarkers and potential therapeutic targets for PCC.
\end{abstract}

Y.-C. Liang, Y.-P. Wu and D.-N. Chen contributed equally to this work. 


\section{Cellular Physiology Cell Physiol Biochem 2018;51:2916-2924 \\ and Biochemistry Published \begin{tabular}{l|l} 
DOI: 10.1159/000496043 & $\begin{array}{l}\text { @ } 2018 \text { The Author(s). Published by S. Karger AG, Basel } \\
\text { www.karger.com/cpb }\end{array}$
\end{tabular}}

Liang et al.: Potential IncRNA Biomarkers for Pheochromocytoma

\section{Introduction}

Pheochromocytoma (PCC) is a neuroendocrine tumor that arises from adrenal medulla or extra-adrenal chromaffin tissues [1, 2]. The annual incidence of PCC is between 3-4 per million [3], and its etiology is not yet clear, but it may be related to genetics. Studies have shown that approximately $30 \%$ of PCC cases have a heredofamilial background $[1,4]$. Due to excessive catecholamine secretion, PCC usually presents a series of symptoms such as hypertension, myocardial infarction, acute respiratory distress syndrome, acute renal failure and cerebrovascular incidents [1]. Due to these serious complications, diagnosing PCC in a timely and effective manner is imperative. Therefore, diagnosis and treatment need to be personalized. This study was designed to find PCC-specific RNAs that could be biomarkers for survival and prognosis.

Recent studies have indicated that $>90 \%$ of the human genome can be transcribed, although only $1 \%-2 \%$ of these genes encode proteins $[5,6]$. Long non-coding RNAs (lncRNAs) are a type of non-coding RNA that encompass a complex variety of biological species. LncRNAs were originally thought to be genomic transcriptional "junk" that were a by-product of RNA polymerase II and without biological function. Nevertheless, recent studies have demonstrated that lncRNAs are involved in genomic imprinting, transcriptional activation, X-inactivation, transcriptional interference, chromatin modification and nuclear transport. LncRNAs also participate in cell differentiation and ontogeny regulation at multiple levels and are closely related to disease states [7-10]. In recent years, research into lncRNAs has progressed rapidly, but the function of most lncRNAs remains unclear. It has been assumed that differentially regulated IncRNAs may be related to survival and prognosis in different cancers, but this has not been directly tested for PCC [11].

Since Salmena et al. put forward the hypothesis of competing endogenous RNAs (ceRNAs) in 2011, we have a deeper understanding of the relationship between RNA interactions. This hypothesis posited that IncRNAs were not a new RNA molecule, but rather a novel regulatory mechanism. Recent studies have shown that genes have multiple modes of action in transcriptional regulation. Some lncRNAs have binding sites with microRNAs and act as miRNA sponges, thus releasing miRNAs from target genes and increasing the expression level of target genes. This mechanism of action is called the ceRNA mechanism [12-15]. Studying the regulatory mechanism of ceRNAs has important clinical significance, such as the analysis of the molecular mechanisms of cancer, and such studies can be of great significance for diagnosis, treatment, and drug development.

Previous studies have shown that ceRNAs play important roles in the occurrence and prognosis of different malignancies. In renal clear cell carcinoma, cell lines that highly express miR- 21 are highly proliferative, while cells that are experimentally manipulated to overexpress PTENP1, which reduces miR-21 levels, show reduced proliferation and invasion [16]. Additionally, ceRNAs have been shown to play tumor suppressive or cancer-promoting role in different contexts. Theoretically, lncRNAs could also play a role in clinical treatment by changing the expression of their corresponding ceRNAs. For example, human epidermal growth factor receptor 2 (HER2) is an important breast cancer prognostic factor, for which Herceptin, a targeted therapeutic for HER2-positive (over-expressed or amplified) has been manufactured [17].

Studies have shown that IncRNAs mainly act as "signal" or "inducible" molecules that bind to proteins and DNA to alter biological function or control gene expression; however, the biological functions of IncRNA in ceRNA networks is varied. Furthermore, a genomewide analysis of ceRNA networks in PCC is lacking. Therefore, we used 183 PCC samples and 3 control samples to establish a ceRNA network based on correlation analysis, which included 23 lncRNAs, 22 mRNAs, and 6 miRNAs. 


\section{Cellular Physiology Cell Physiol Biochem 2018;51:2916-2924 and Biochemistry \begin{tabular}{l|l} 
DOI: 10.1159/000496043 & $\begin{array}{l}\text { (c) } 2018 \text { The Author(s). Published by S. Karger AG, Basel } \\
\text { www.karger.com/cpb }\end{array}$
\end{tabular}}

Liang et al.: Potential IncRNA Biomarkers for Pheochromocytoma

\section{Materials and Methods}

Patient information from The Cancer Genome Atlas (TCGA) dataset

We included a total of 183 PCC samples and 3 control samples for further analysis. All data were derived from TCGA database, including clinical sample information, miRNAseq data, lncRNAseq data, and mRNASeq data. We used the Data Transfer Tool to download the data, which contained numerous independent data files, each of which was specific for one sample. Sequencing data was converted through Homo_sapiens. GRCh38.87.chr.gtf.

Analysis of differentially expressed IncRNAs, mRNAs, and miRNAs

We used Empirical Analysis of Digital Gene Expression Data package in R (edgeR) to analyze differentially expressed RNAs (DERNAs) [18]; differentially expressed mRNAs (DEmRNAs), IncRNAs (DElncRNAs) and miRNAs (DEmiRNAs) were evaluated with the cut-off criteria of false discovery rate (FDR; adjusted $p$ value) $<0.01$ and $\mid \log 2$ fold Change (FC) $\mid>2.0$ [19]. Then, we selected the intersection of differentially expressed PCC IncRNAs, mRNAs and miRNAs for subsequent analysis.

\section{Biological processes and pathways functional enrichment analysis}

We employed the Database for Annotation, Visualization, and Integrated Discovery (DAVID) to obtain information for Gene Ontology (GO) biological processes and the Kyoto Encyclopedia of Genes and Genomes (KEGG) pathways. The selection criteria were set as $\mathrm{P}<0.05$ and an enrichment score $>1.5$.

\section{Constructing the ceRNA network}

According to previous studies, we know that IncRNAs can affect mRNAs through miRNAs. Based on this theory, we built a ceRNA network through three steps: 1) we used miRcode (http://www.mircode. org/) to analyze interactions between lncRNAs and miRNAs based on the differential expression of PCC miRNA; 2) we used miRanda (http://www.microrna.org/microrna/home.do), miRDB (http://www.mirdb. org/) and TargetScan (http://www.targetscan.org/) to predict miRNA-targeted mRNA; and 3) we kept the intersection of the differentially expressed IncRNAs and mRNAs, and used Cytoscape v3.0 to establish the lncRNA/mRNA/miRNA ceRNA network.

\section{Survival analysis}

We used the "survival" package in R to determine survival curves, which were stratified by the patients' prognostic value of DERNAs, including DEmRNAs, DEmiRNAs and DElncRNAs. The clinical data for these PCC patients came from TCGA. Kaplan-Meier curves were used to evaluate univariate survival analysis. P-values $<0.05$ were considered statistically significant.

\section{Results}

Identifying DERNAs in primary tumor and control samples

We compared the normal samples with PCC samples, and in total, 554 DElncRNAs, 1775 DEmRNAs and 40 DEmiRNAs were selected by edgeR. Then heat maps on all linkage clustering of DEIncRNA, DEmRNAs and DEmiRNAs were constructed by the "gplots" package in R (Supplementary Fig. S1 - for all supplementary material see www.karger-com/ doi/10.1159/000496043). Consequently, there were 327 (59.03\%) downregulated and 227 (40.97\%) upregulated DElncRNAs, 21 (52.5\%) downregulated and 19 (47.5\%) upregulated DEmiRNAs and 779 (43.89\%) downregulated and 996 (56.11\%) upregulated DEmRNAs.

\section{GO terms and KEGG pathways for differentially expressed $m R N A s$}

To further study the function of these differentially expressed genes, we selected 1775 differentially expressed mRNAs for further analysis and show the top $10 \mathrm{GO}$ biological processes and 10 KEGG pathways. The biological function of these genes was mainly enriched in neurotransmitter secretion, and there was also an enrichment of intracellular signal transduction and nervous system development. Finally, KEGG pathway analyses 


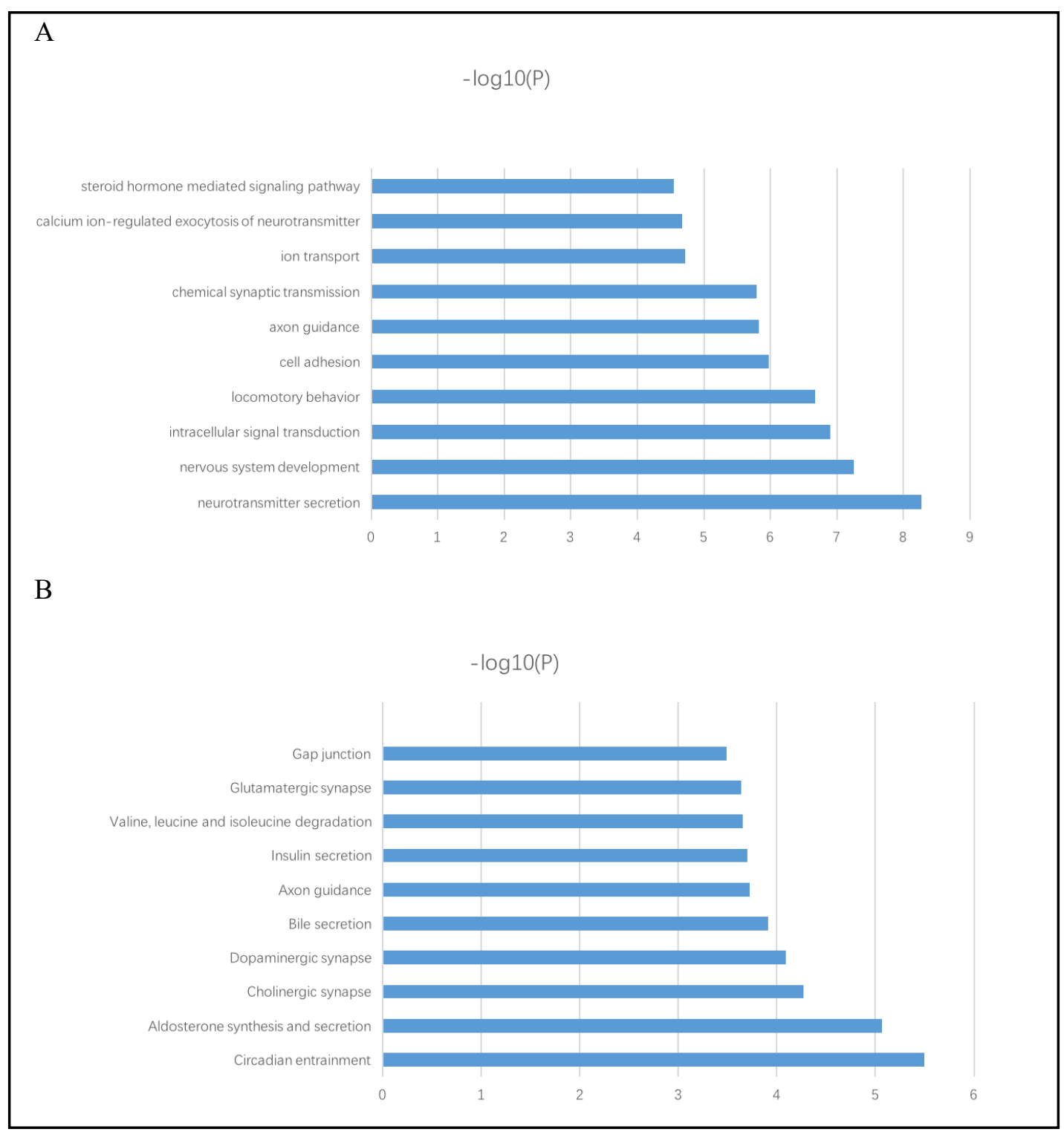

Fig. 1. Top 10 GO terms (A) and KEGG pathways (B) for differentially expressed mRNAs in primary tumor and control samples.

demonstrate that the most paramount pathways were circadian entrainment, aldosterone synthesis and secretion, and cholinergic synapse (Fig. 1).

CeRNA network construction in PCC

We employed miRcode to analyze interactions between IncRNAs and miRNAs and found that 6 of 40 PCC-specific miRNAs might target to the 23 of 554 PCC-restricted lncRNAs (Table 1). Then we used miRanda, miRDB and TargetScan to predicted these 6 miRNA-targeted mRNAs to explore the relationship between miRNAs and mRNAs. The results indicated that only 5 PCC-specific miRNAs might target 22 of the 1775 PCC-specific mRNAs (Table 2). MiRNA-targeted mRNAs were discarded if they were not included in DERNAs. Based on these data (Table 1 and 2), we used Cytoscape 3.0 to construct the IncRNA-miRNA-mRNA ceRNA network. Finally, 23 lncRNAs, 22 mRNAs, and 6 miRNAs were included in the ceRNA network (Fig. 2). 


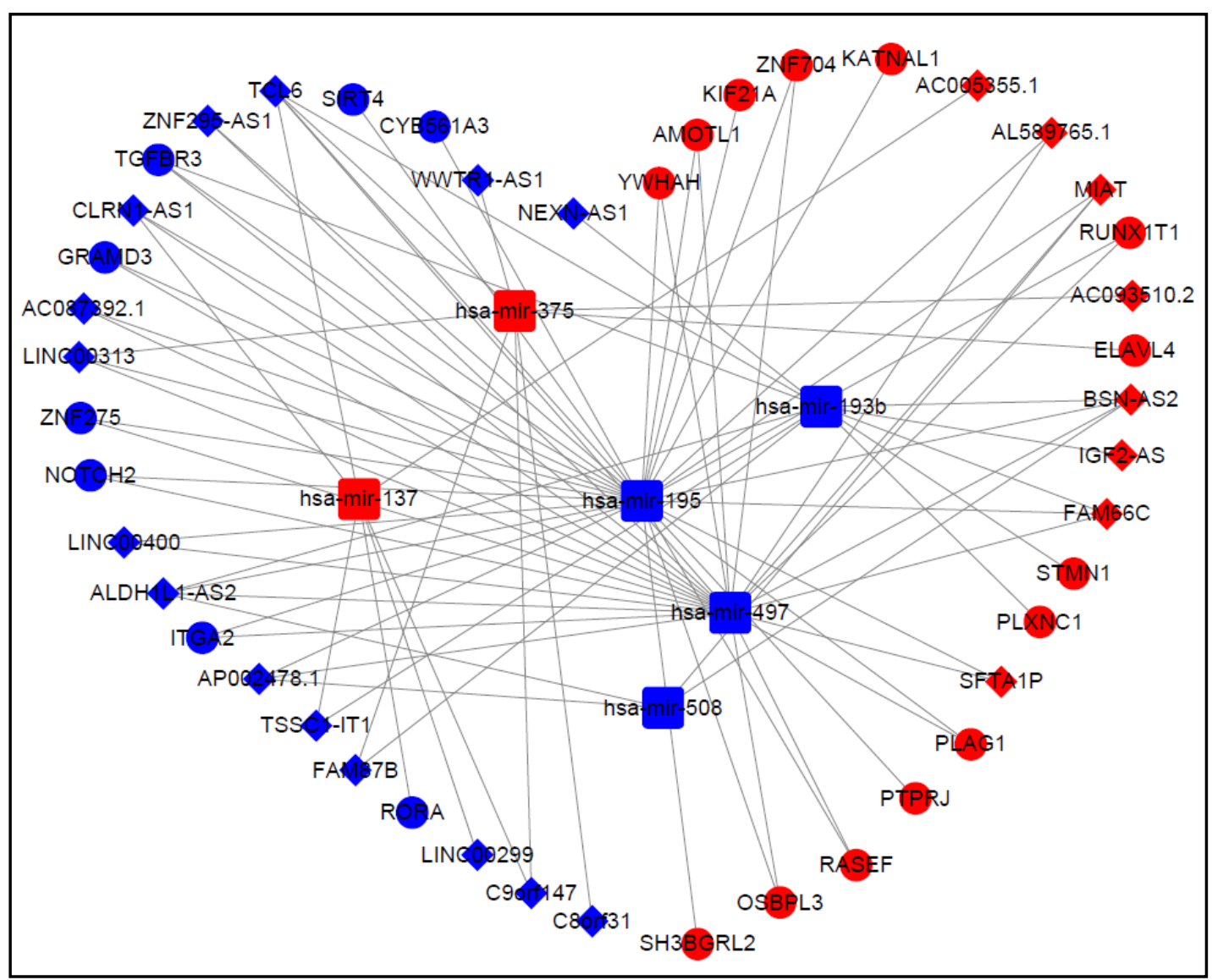

Fig. 2. CeRNA network in pheochromocytoma. Red nodes represent a high level of expression, while blue nodes represent a low level of expression. Ellipses represent protein-coding genes; rectangles represent miRNAs; diamonds represent lncRNAs; gray edges indicate IncRNA-miRNA-mRNA interactions.

Table 1. The MiRcode database revealed interactions between 23 DElncRNAs and 6 DEmiRNAs

\begin{tabular}{lc}
\hline IncRNA & miRNA \\
\hline AC005355.1, C9orf147, CLRN1-AS1, LINC00299, TCL6, TSSC1-IT1 & hsa-mir-137 \\
IGF2-AS, ALDH1L1-AS2, BSN-AS2, FAM66C, FAM87B, NEXN-AS1, TCL6, TSSC1-IT1 & hsa-mir-193b \\
AC087392.1, AL589765.1, ALDH1L1-AS2, AP002478.1, BSN-AS2, CLRN1-AS1, FAM66C, LINC00313, & hsa-mir-195 \\
LINC00400, MIAT, SFTA1P, TCL6, ZNF295-AS1 & hsa-mir-375 \\
AC093510.2, C8orf31, C9orf147, FAM87B, LINC00313, TCL6, WWTR1-AS1 & hsa-mir-497 \\
AC087392.1, AL589765.1, ALDH1L1-AS2, AP002478.1, BSN-AS2, CLRN1-AS1, FAM66C, LINC00313, & hsa-mir-508 \\
\hline
\end{tabular}

\section{Survival analysis of key DERNAs in PCC}

We next analyzed the correlation of overall survival (OS) with DERNAs in the ceRNA network, with OS for all DERNAs studied using the Kaplan--Meier curve analysis to evaluate patient prognostic potential. We discovered that two of 23 DElncRNAs were considered key DERNAs that were related to the survival prognosis of PCC. Consequently, 2 DElncRNAs were significantly associated with OS, and the IncRNAs C9orf147 and BSN-AS2 were negatively associated with OS ( $\log$-rank P $<0.05$; Fig. 3). Similar to the DElncRNAs, we also used KaplanMeier curve analysis to determine the OS for 22 DEmRNAs in PCC. Two of the 22 DEmRNAs were related to OS (log-rank P <0.05). mRNA PTPRJ was found to be associated with low 
Table 2. The MiRcode database revealed interactions between five DEmiRNAs and 22 DEmRNAs

\begin{tabular}{lc}
\hline miRNA & mRNA \\
\hline hsa-mir-137 & RORA \\
hsa-mir-193b & PLXNC1, STMN1, TGFBR3 \\
hsa-mir-195 & TGFBR3, NOTCH2, ZNF704, CYB561A3, AMOTL1, KATNAL1, YWHAH, PLAG1, KIF21A, SIRT4, \\
hsa-mir-375 & GRAMD3, ZNF275, ITGA2, OSBPL3, SH3BGRL2, PTPRJ, RUNX1T1, RASEF \\
hsa-mir-497 & ELAVL4 \\
& YWHAH, ZNF275 \\
\hline
\end{tabular}

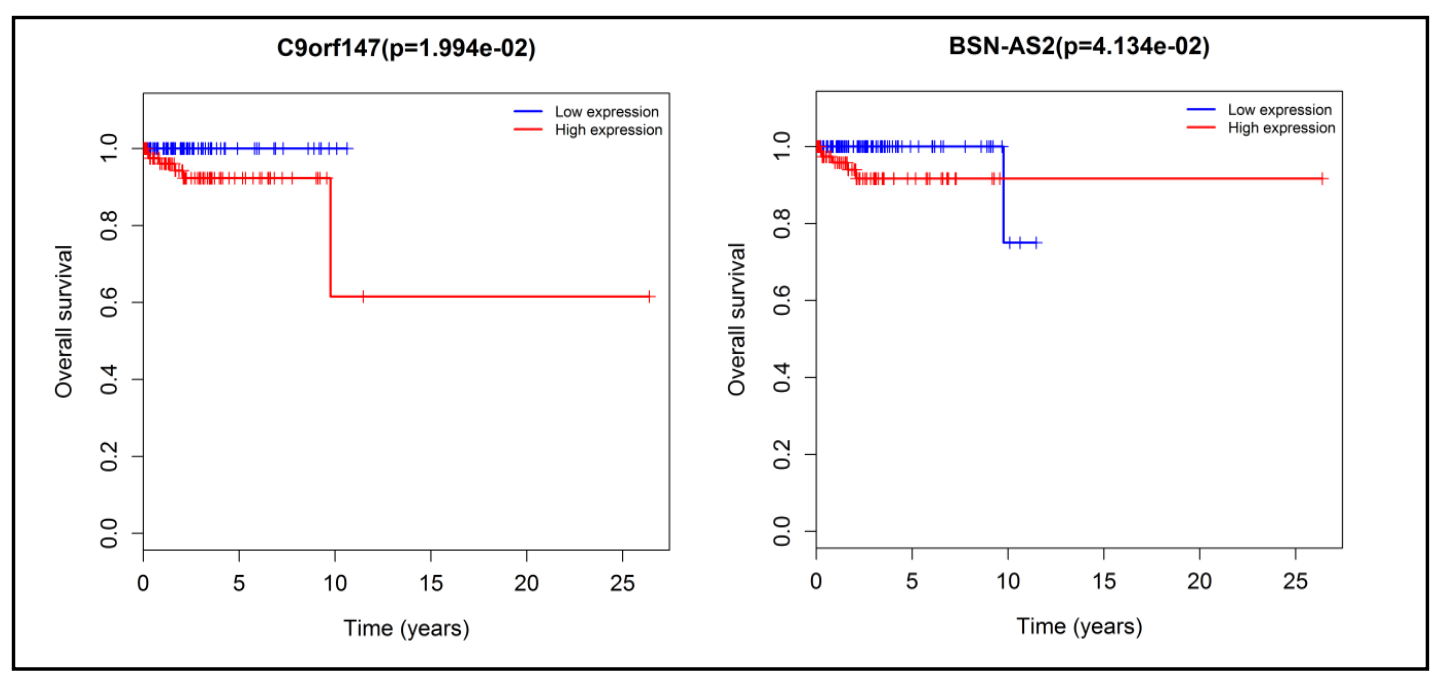

Fig. 3. Kaplan-Meier survival curves for two lncRNAs associated with overall survival in pheochromocytoma. Two DElncRNAs are presented: C9orf147 and BSN-AS2 $(\mathrm{P}<0.05)$.

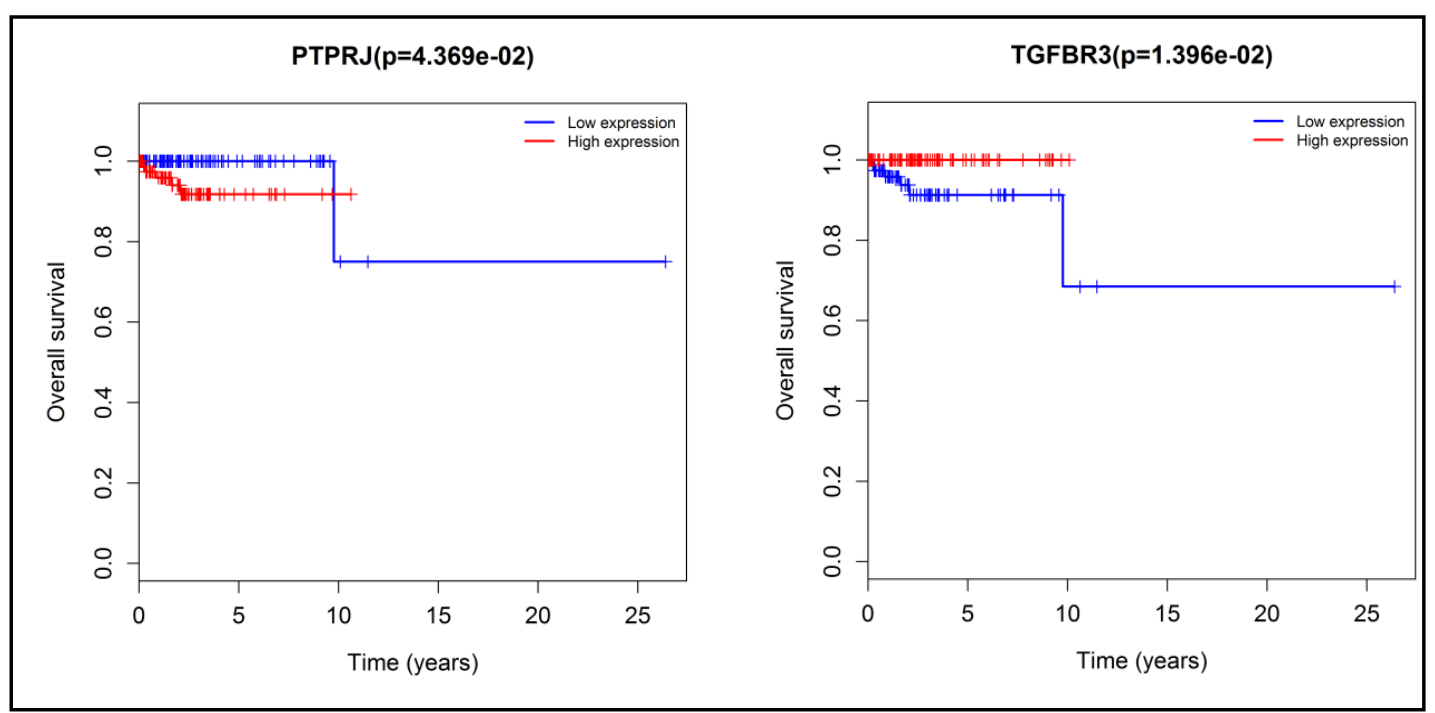

Fig. 4. Kaplan-Meier survival curves for two protein-coding genes associated with overall survival in pheochromocytoma. Two DEmRNAs are presented: PTPRJ and TGFBR3 $(\mathrm{P}<0.05)$.

levels of DEmRNAs and with good prognosis, while higher expression of mRNA TGFBR3 was associated with a better prognosis (Fig. 4). 


\section{Cellular Physiology Cell Physiol Biochem 2018;51:2916-2924

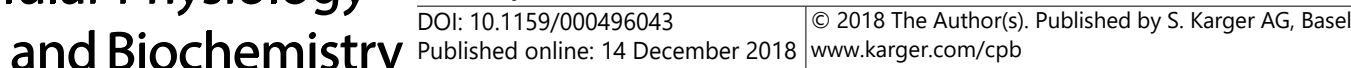

Liang et al.: Potential IncRNA Biomarkers for Pheochromocytoma

\section{Discussion}

In recent years, accumulating evidence has show that the mode of mutual regulation between IncRNAs and miRNAs and their downstream target genes is closely related to the occurrence and development of tumors; thus, this has become a hot spot in the field of cancer research. miRNAs are an important factor for the post-transcriptional regulation of genes, and their activity can be controlled by lncRNAs, which are absorbed by "sponges"[12].

The more miRNA binding sites on a IncRNA, the stronger the competition [20]. When lncRNAs are expressed at low levels, they can only bind to a small fraction of the miRNAs. In this situation, a large number of remaining miRNAs can bind to the target mRNA and promote its degradation. This new regulatory model of IncRNA-miRNA-mRNA complements the previous traditional miRNA $\rightarrow$ mRNA gene regulation model [12].

In addition to being directly involved in the regulation of gene expression, lncRNAs can also interact with miRNAs as ceRNAs and participate in the regulation of target gene expression. Thus, this also plays an important role in tumor cell proliferation, apoptosis, angiogenesis, invasion and metastasis [21-23]. Some studies have shown that differentially expressed IncRNAs are significantly associated with the prognosis of tumors and can be used as tumor-related predictors $[24,25]$. Yang et al. investigated the role of IncRNA MALAT-1 in PC-12 cells, and found that MALAT- 1 can promote oxidative stress and apoptosis by activating the p38MAPK pathway in this cell line [26]. Zang et al. demonstrated that downregulation of IncRNA BC088414 leads to a significant inhibition of apoptosis in pheochromocytoma cells through Adrb2 and CASP6[27]. Tian et al. reported that downregulating lncRNA UCA1 resulted in a significant inhibition of cell viability, invasion and a reduction in the migration of PC-12 cell by reducing, in combination with Mir-18a, the expression of SOX6 target genes [28].

In this study, we analyzed the differentially expressed IncRNAs, miRNAs and mRNAs in PCC compared with non-tumor and tumor tissues. We further analyzed the function and pathways of PCC-specific mRNAs using the GO and KEGG analysis programs. GO revealed that the functions of differentially regulated genes were mainly enriched in neurotransmitter secretion, intracellular signal transduction and nervous system development. The results of the KEGG pathway analysis revealed that most of the differentially expressed genes were associated with hormone secretion and regulation. Circadian entrainment signaling plays crucial roles in reducing cellular function, affecting cell cycle, apoptosis and the expression of genes involved in various other pathways, thereby potentially increasing the possibility of disease, including cancer $[29,30]$. The aldosterone synthesis and secretion pathway substantially impacts myocardial hyperplasia and fibrosis, induces dysfunction of vascular endothelial cells and promotes vascular remodeling [31]. The gap junction pathway also plays crucial roles in coordinating intercellular homeostasis, stimulating differentiation and cell growth, and increasing the transmission of information through nerve impulses [32, 33]. These functions and pathways are closely related to the occurrence and development of PCC.

Using the ceRNA network in PCC, we further analyzed the potential mechanism of the intersections among lncRNAs, miRNAs and mRNAs in PCC. In our study, 23 DElncRNAs were identified in PCC compared with normal samples. The results showed that two DElncRNAs were significantly related to OS, IncRNA C9orf147 and BSN-AS2. However, there are no reports of these two lncRNAs in other diseases. Additionally, no study has investigated the function of C9orf147 or BSN-AS2. These IncRNAs may play important roles in PCC pathogenesis and act as prognostic markers. BSN-AS2 expression was upregulated in 183 PCC samples compared with the 3 control samples. We found that high BSN-AS2 expression was associated with poor prognosis in PCC. According to the ceRNA network we built, BSN-AS2 may adjust PTPRJ mRNA expression through miR-195, and PTPRJ expression was negatively correlated with OS. Conversely, BSN-AS2 may also compete with miR-193b, miR-195 and/ or miR-497 to mediate TGFBR3 mRNA expression, which was positively associated with OS. However, TGFBR3 expression was downregulated in PCC, thus, there may be other factors 
regulating its expression. Furthermore, we observed that low IncRNA C9orf147 expression was associated with a good prognosis in PCC.

Although this study might have significant clinical significance, we still need to consider several limitations. First, the sample number of the control group was very small, although it has existed in previous studies. Second, our data came from TCGA database, which may include selection bias. Information including benign and malignant, unilateral and bilateral, hereditary and sporadic diseases could not be distinguished based on data extracted from TCGA database. Thus, further study could not be made in terms of distinguish the differences of long non-coding RNA biomarkers and the gene expression heterogeneity between those details. Third, the function of C9orf147 and BSN-AS2 in PCC needs to be further studied in vivo and in vitro.

\section{Conclusion}

In conclusion, this study revealed PCC-specific lncRNAs that could be prognostic markers for PCC patients using bioinformatics analysis from TCGA database. Additionally, by building a ceRNA network with PCC-specific IncRNAs, miRNAs and mRNAs, we clarified the mechanism of PCC at the genetic level better and elucidated the relationship between these three RNA species. Our research increases the understanding of the pathogenesis of PCC and offers novel IncRNAs as underlying therapeutic targets or prognostic biomarkers.

\section{Disclosure Statement}

All authors declare that they have no conflicts of interest.

\section{References}

1 Lenders JW, Eisenhofer G, Mannelli M, Pacak K: Phaeochromocytoma. Lancet 2005;366:665-675.

2 Bravo EL, Gifford RW, Jr.: Current concepts. Pheochromocytoma: diagnosis, localization and management. N Engl J Med 1984;311:1298-1303.

3 Fung MM, Viveros OH, O'Connor DT: Diseases of the adrenal medulla. Acta Physiol (Oxf) 2008;192:325335.

-4 Amar L, Bertherat J, Baudin E, Ajzenberg C, Bressac-de Paillerets B, Chabre O, Chamontin B, Delemer B, Giraud S, Murat A, Niccoli-Sire P, Richard S, Rohmer V, Sadoul JL, Strompf L, Schlumberger M, Bertagna $\mathrm{X}$, Plouin PF, Jeunemaitre X, Gimenez-Roqueplo AP: Genetic testing in pheochromocytoma or functional paraganglioma. J Clin Oncol 2005;23:8812-8818.

-5 Xiao K, Yu Z, Li X, Li X, Tang K, Tu C, Qi P, Liao Q, Chen P, Zeng Z, Li G, Xiong W: Genome-wide Analysis of Epstein-Barr Virus (EBV) Integration and Strain in C666-1 and Raji Cells. J Cancer 2016;7:214-224.

6 Xu K, Xiong W, Zhou M, Wang H, Yang J, Li X, Chen P, Liao Q, Deng H, Li X, Li G, Zeng Z: Integrating ChIPsequencing and digital gene expression profiling to identify BRD7 downstream genes and construct their regulating network. Mol Cell Biochem 2016;411:57-71.

7 Ponting CP, Oliver PL, Reik W: Evolution and functions of long noncoding RNAs. Cell 2009;136:629-641.

-8 Maeda N, Kasukawa T, Oyama R, Gough J, Frith M, Engstrom PG, Lenhard B, Aturaliya RN, Batalov S, Beisel KW, Bult CJ, Fletcher CF, Forrest AR, Furuno M, Hill D, Itoh M, Kanamori-Katayama M, Katayama S, Katoh M, Kawashima T et al.: Transcript annotation in FANTOM3: mouse gene catalog based on physical cDNAs. PLoS Genet 2006;2:e62.

-9 Djebali S, Davis CA, Merkel A, Dobin A, Lassmann T, Mortazavi A, Tanzer A, Lagarde J, Lin W, Schlesinger F, Xue C, Marinov GK, Khatun J, Williams BA, Zaleski C, Rozowsky J, Roder M, Kokocinski F, Abdelhamid RF, Alioto T et al.: Landscape of transcription in human cells. Nature 2012;489:101-108. 


\section{Cellular Physiology Cell Physiol Biochem 2018;51:2916-2924

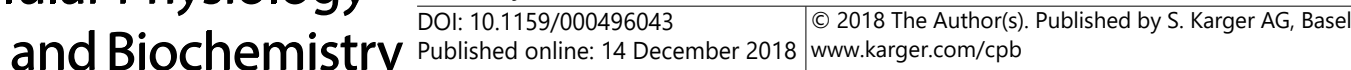

Liang et al.: Potential IncRNA Biomarkers for Pheochromocytoma

10 Guttman M, Amit I, Garber M, French C, Lin MF, Feldser D, Huarte M, Zuk O, Carey BW, Cassady JP, Cabili MN, Jaenisch R, Mikkelsen TS, Jacks T, Hacohen N, Bernstein BE, Kellis M, Regev A, Rinn JL, Lander ES: Chromatin signature reveals over a thousand highly conserved large non-coding RNAs in mammals. Nature 2009;458:223-227.

11 Sui J, Xu SY, Han J, Yang SR, Li CY, Yin LH, Pu YP, Liang GY: Integrated analysis of competing endogenous RNA network revealing lncRNAs as potential prognostic biomarkers in human lung squamous cell carcinoma. Oncotarget 2017;8:65997-66018.

12 Salmena L, Poliseno L, Tay Y, Kats L, Pandolfi PP: A ceRNA hypothesis: the Rosetta Stone of a hidden RNA language? Cell 2011;146:353-358.

13 Ebert MS, Neilson JR, Sharp PA: MicroRNA sponges: competitive inhibitors of small RNAs in mammalian cells. Nat Methods 2007;4:721-726.

14 Poliseno L, Salmena L, Zhang J, Carver B, Haveman WJ, Pandolfi PP: A coding-independent function of gene and pseudogene mRNAs regulates tumour biology. Nature 2010;465:1033-1038.

15 Bartel DP: MicroRNAs: target recognition and regulatory functions. Cell 2009;136:215-233.

-16 Yu G, Yao W, Gumireddy K, Li A, Wang J, Xiao W, Chen K, Xiao H, Li H, Tang K, Ye Z, Huang Q Xu H: Pseudogene PTENP1 functions as a competing endogenous RNA to suppress clear-cell renal cell carcinoma progression. Mol Cancer Ther 2014;13:3086-3097.

17 Ow TJ, Sandulache VC, Skinner HD, Myers JN: Integration of cancer genomics with treatment selection: from the genome to predictive biomarkers. Cancer 2013;119:3914-3928.

18 Robinson MD, McCarthy DJ, Smyth GK: edgeR: a Bioconductor package for differential expression analysis of digital gene expression data. Bioinformatics 2010;26:139-140.

19 Qiu J, Zhang W, Xia Q, Liu F, Li L, Zhao S, Gao X, Zang C, Ge R, Sun Y: RNA sequencing identifies crucial genes in papillary thyroid carcinoma (PTC) progression. Exp Mol Pathol 2016;100:151-159.

-20 Hansen TB, Jensen TI, Clausen BH, Bramsen JB, Finsen B, Damgaard CK, Kjems J: Natural RNA circles function as efficient microRNA sponges. Nature 2013;495:384-388.

-21 Wang J, Liu X, Wu H, Ni P, Gu Z, Qiao Y, Chen N, Sun F, Fan Q: CREB up-regulates long non-coding RNA, HULC expression through interaction with microRNA-372 in liver cancer. Nucleic Acids Res 2010;38:5366-5383.

22 Liu XH, Sun M, Nie FQ, Ge YB, Zhang EB, Yin DD, Kong R, Xia R, Lu KH, Li JH, De W, Wang KM, Wang ZX: Lnc RNA HOTAIR functions as a competing endogenous RNA to regulate HER2 expression by sponging miR331-3p in gastric cancer. Mol Cancer 2014;13:92.

23 Zhou X, Gao Q Wang J, Zhang X, Liu K, Duan Z: Linc-RNA-RoR acts as a "sponge" against mediation of the differentiation of endometrial cancer stem cells by microRNA-145. Gynecol Oncol 2014;133:333-339.

24 Augoff K, McCue B, Plow EF, Sossey-Alaoui K: miR-31 and its host gene lncRNA LOC554202 are regulated by promoter hypermethylation in triple-negative breast cancer. Mol Cancer 2012;11:5.

25 Li H, Yu B, Li J, Su L, Yan M, Zhu Z, Liu B: Overexpression of IncRNA H19 enhances carcinogenesis and metastasis of gastric cancer. Oncotarget 2014;5:2318-2329.

-26 Yang L, Xu F, Zhang M, Shang XY, Xie X, Fu T, Li JP, Li HL: Role of LncRNA MALAT-1 in hypoxia-induced PC12 cell injury via regulating p38MAPK signaling pathway. Neurosci Lett 2018;670:41-47.

-27 Zhao FY, Tang J, Zhang L, Li SP, Feng Y, Liu HT, Qu Y, Mu DZ: [Role of long non-coding RNA BC088414 in hypoxic-ischemic injury of neural cells]. Zhongguo Dang Dai Er Ke Za Zhi 2015;17:1348-1353.

28 Tian J, Xu H, Chen G, Wang H, Bi Y, Gao H, Luo Y: Roles of IncRNA UCA1-miR-18a-SOX6 axis in preventing hypoxia injury following cerebral ischemia. International Journal of Clinical \& Experimental Pathology 2017;10:8187-8198.

-29 Hoffman AE, Yi CH, Zheng T, Stevens RG, Leaderer D, Zhang Y, Holford TR, Hansen J, Paulson J, Zhu Y: CLOCK in breast tumorigenesis: genetic, epigenetic, and transcriptional profiling analyses. Cancer Res 2010;70:1459-1468.

30 Fu L, Lee CC: The circadian clock: pacemaker and tumour suppressor. Nat Rev Cancer 2003;3:350-361.

31 Schiffrin EL: Effects of aldosterone on the vasculature. Hypertension 2006;47:312-318.

-32 Kumar NM, Gilula NB: The gap junction communication channel. Cell 1996;84:381-388.

-33 Christ GJ: Modulation of alpha 1-adrenergic contractility in isolated vascular tissues by heptanol: a functional demonstration of the potential importance of intercellular communication to vascular response generation. Life Sci 1995;56:709-721. 